\title{
Electroacupuncture improves acute bowel injury recovery in rat models
}

\author{
JIANNONG WU ${ }^{1}$, BIN LYU $^{2}$, TIE'ER GAN $^{3}$, LINGCONG WANG $^{1}$ and MEIFEI ZHU ${ }^{1}$ \\ ${ }^{1}$ Department of Critical Care; ${ }^{2}$ Division of Gastroenterology; ${ }^{3}$ Department of Infectious Diseases, The First Affiliated \\ Hospital, Zhejiang University of Traditional Chinese Medicine, Hangzhou, Zhejiang 310053, P.R. China
}

Received October 19, 2015; Accepted February 24, 2017

DOI: 10.3892/etm.2017.5159

\begin{abstract}
Electroacupuncture (EA) accelerates intestinal functional recovery in sepsis. The present study investigated ghrelin and ghrelin receptor (GSH-R) levels during EA in rats with acute bowel injury (ABI). Rats were grouped into four groups: Sham, $\mathrm{ABI}, \mathrm{ABI}+\mathrm{EA}$ and $\mathrm{ABI}+\mathrm{GHRA}+\mathrm{EA}$ ( $\mathrm{n}=12$ per group). $\mathrm{ABI}$ was induced by cecal ligation and puncture (CLP). EA on bilateral Zusanli acupoints was performed following CLP. GSH-R blocker (GHRA) was used following CLP but prior to EA for $\mathrm{ABI}+\mathrm{GHR} A+\mathrm{EA}$ rats. Rats were sacrificed $12 \mathrm{~h}$ following CLP. Serum ghrelin, tumor necrosis factor- $\alpha(\mathrm{TNF}-\alpha)$ and high mobility group box 1 (HMGB1) levels, as well as ghrelin and GSH-R protein expression, water content, pathological changes and myeloperoxidase (MPO) and diamine oxidase (DAO) activities in the bowel tissues, were measured. ABI rats, compared with the sham rats, had significantly lower levels of ghrelin and GSH-R in the serum and bowel tissue, and higher Chiu's score (all $\mathrm{P}<0.05$ ). The ABI+EA rats, compared with the ABI rats, had significantly reduced serum TNF- $\alpha$ and HMGB1 levels, bowel water content, MPO activity and Chiu's score (all $\mathrm{P}<0.05$ ), and significantly higher serum ghrelin $(121.2 \pm 10.7 \mathrm{vs} .86 .7 \pm 6.4 \mathrm{pg} / \mathrm{ml})$, bowel ghrelin $(0.12 \pm 0.02$ vs. $0.08 \pm 0.01)$, GSH-R $(0.05 \pm 0.04$ vs $0.03 \pm 0.01)$ and DAO activity $(18.74 \pm 4.18$ vs. $13.52 \pm 2.33 \mathrm{U} / \mathrm{ml}$; all $\mathrm{P}<0.05)$, indicating an improvement of the intestinal mucosal barrier. GHRA reversed the protective effects of EA. Therefore, EA improved ABI recovery by promoting ghrelin secretion and upregulating GSH-R expression.
\end{abstract}

\section{Introduction}

Acute bowel injury (ABI) is a condition caused by bowel ischemia, edema and increased permeability resulting from

Correspondence to: Dr Bin Lyu, Division of Gastroenterology, The First Affiliated Hospital, Zhejiang University of Traditional Chinese Medicine, 54 Post and Telecommunication Road, Hangzhou, Zhejiang 310053, P.R. China

E-mail: 1vbin@medmail.com.cn

Key words: sepsis, acute bowel injury, cecal ligation and puncture, electroacupuncture, ghrelin, ghrelin receptor sepsis, trauma or major surgery, which is also referred to as acute intestinal distress syndrome (1). Increased intestinal permeability in ABI results in the translocation of large amounts of intestinal bacteria, thus initiating or aggravating systematic inflammatory response syndrome. ABI may lead to the development of multiple organ dysfunction syndrome (MODS) (2). Current treatment for sepsis to prevent MODS requires rapid diagnosis to allow timely treatment with antibiotics and the required interventions, such as resuscitation (3). Therefore, early and effective inhibition of the production of pro-inflammatory factors may protect against the intestinal damage caused by excessive inflammation, in addition to preventing and treating sepsis and MODS.

A number of studies undertaken in China have demonstrated that electroacupuncture on Zusanli acupoints significantly decreases levels of tumor necrosis factor- $\alpha$ (TNF- $\alpha)$ in the liver, kidney and jejunum tissues in rat models of ABI $(4,5)$. Electroacupuncture may improve blood flow and perfusion of the tissues, thus reducing organ edema and dysfunction (6). In a previous clinical study, it was demonstrated that electroacupuncture on Zusanli acupoints significantly reduces the urinary lactulose/mannitol (L/M) ratio and levels of plasma D-lactic acid. This suggests it may improve the function of the intestinal mucosal barrier, thus decreasing intestinal permeability. It was demonstrated that electroacupuncture accelerated intestinal function recovery and achieved early target feeding in sepsis patients. In addition, serum levels of inflammatory factors, including C-reactive protein, interleukin (IL)-6 and TNF- $\alpha$, were significantly reduced, as was the APACHE II score, while immune functions significantly improved (7).

Previous studies have indicated that ghrelin may improve the intestinal mucosal barrier in patients with sepsis $(8,9)$. Ghrelin is an endogenous brain-gut peptide primarily secreted by endocrine cells in gastric mucosa (primarily gastric $\mathrm{X} / \mathrm{A}$-like cells) and serves as an endogenous ligand for the growth hormone secretagogue receptor (GHS-R). Ghrelin has multiple physiological functions. It increases appetite, stimulates the secretion of growth hormone $(\mathrm{GH})$, promotes fat deposition, increases gastrointestinal motility and increases gastric acid secretion. Ghrelin therefore promotes growth, development and weight gain (10). Furthermore, ghrelin inhibits the production of pro-inflammatory factors including TNF- $\alpha$, IL- 6 and high mobility group box 1 (HMGB1) and protects organ function in patients with sepsis $(9,11,12)$. 
The present study hypothesized that the mechanisms involved in the protective effects of electroacupuncture on ABI may be associated with the increased secretion of ghrelin and upregulated expression of GSH-R. Therefore, in the present study, cecal ligation and puncture (CLP) was performed to produce septic ABI rat models, electroacupuncture on Zusanli acupoints was conducted and TNF- $\alpha$ and HMGB1 levels in the serum were measured. Additionally, myeloperoxidase (MPO) and diamine oxidase (DAO) activities, water content and pathological changes in the bowel tissues were measured to evaluate the protective effects of electroacupuncture on Zusanli acupoints on ABI. In addition, changes in the ghrelin and GHS-R levels in serum and bowel tissues were also evaluated to investigate the mechanisms involved in the protective effects of electroacupuncture on ABI, and thus provide theoretical basis for the clinical treatment of $\mathrm{ABI}$ and further experimental studies. The results suggest that electroacupuncture decreases the production of TNF- $\alpha$ and HMGB1, which are increased in ABI. This protective process involves increased ghrelin expression and is inhibited by the blocking of GHS-R.

\section{Materials and methods}

Animals. Male specific pathogen free (SPF) level Sprague-Dawley rats $(\mathrm{n}=48)$ between 10 and 12 -weeks old, with a body weight of $295 \pm 22 \mathrm{~g}$, were obtained from Shanghai Sippr BK laboratory animals, Ltd. (Shanghai China; license number, SCXK[Hu]2008-0016). All rats were acclimatized in housing conditions at $25 \pm 2^{\circ} \mathrm{C}, 55 \pm 2 \%$ humidity and a $12 \mathrm{~h}$ light/dark cycle. Rats were fed with conventional laboratory feed and provided with water ad libitum. Following acclimatization, rats were fasted for $12 \mathrm{~h}$ and water fasted for $4 \mathrm{~h}$ prior to surgery. All procedures and animal experiments were approved by the Animal Care and Use Committee of Zhejiang Chinese Medical University (No. 2014-K-183-01).

A random number table method was used to assign the rats into 4 groups ( $n=12$ each group): Sham group, $A B I$ group, $\mathrm{ABI}+$ electroacupuncture (EA) group, and $\mathrm{ABI}+\mathrm{GHRA}+\mathrm{EA}$ group.

Acute bowel injury rat models induced by cecal ligation and puncture. $\mathrm{ABI}$ rat models induced by severe abdominal infection were induced according to standard cecal ligation and puncture (CLP) methods $(13,14)$. The rats were anesthetized by intraperitoneal injection of $3 \%$ sodium pentobarbital solution $(45 \mathrm{mg} / \mathrm{kg}$, Chinese Pharmaceutical Group Shanghai Chemical Reagent Company, Shanghai, China), aseptic operation was performed to make an incision along the midline of the abdomen. When the cecum was exposed and the root was ligated (to avoid the ligation of the blood vessels at the root of ileum and cecum), a size 16 needle was used to puncture the cecum 4 times, then the cecum was put back into the abdominal cavity and the abdominal incision was closed layer by layer. For the rats in the Sham group, the incision along the midline of the abdomen was made and closed immediately.

Electroacupuncture intervention. For the rats in the ABI+EA group, electroacupuncture was performed 20 min following CLP. In brief, electroacupuncture was performed by $7 \mathrm{~mm}$ perpendicular insertion of a needle on the bilateral Zusanli acupoints $(5 \mathrm{~mm}$ below the capitula fibula, posterior-lateral side of the knee joint), then an electroacupuncture apparatus (G6805-I; Qingdao Xinsheng Industry Co., Ltd., Qingdao, China) was connected to the needle and rats stimulated for 30 min with a current of $3 \mathrm{~mA}$ and frequency of $2-100 \mathrm{~Hz}$.

For the rats in the ABI+GHR A+EA group, a micro-injection pump (Shanghai Alcott biotech Co., Ltd., Shanghai, China) was used to inject GSH-R blocker [D-Arg ${ }^{1}, \mathrm{D}-\mathrm{Phe}^{5}, \mathrm{D}-\mathrm{Trp}^{7.9}$, $\mathrm{Leu}^{11}$ ] (Shanghai Absin Bioscience, Inc., Shanghai, China) substance $\mathrm{P}(700 \mathrm{ng} / \mathrm{kg}$, diluted with $0.9 \% \mathrm{NaCl}$ to obtain a solution of $1 \mathrm{ml}$ ) for $1 \mathrm{~h}$ at the tail vein. Electroacupuncture on Zusanli acupoints was performed 20 min following the injection following the same procedure as used for the ABI+EA group.

Specimen collection. For rats in each group, blood was collected $12 \mathrm{~h}$ following CLP. The blood was centrifuged at $4^{\circ} \mathrm{C}$ at $380 \mathrm{x}$ g for $20 \mathrm{~min}$ and the serum was collected and stored at $-80^{\circ} \mathrm{C}$ until examination of serum TNF- $\alpha$, HMGB1 and ghrelin levels. Prior to sacrifice, the 48 rats were anesthetized by intraperitoneal injection of 3\% sodium pentobarbital solution $(45 \mathrm{mg} / \mathrm{kg})$. Rats were sacrificed by cervical dislocation immediately following blood collection. The abdomen was incised and bowel tissue $\sim 3 \mathrm{~cm}$ in length was quickly collected $2,5,8$, and $11 \mathrm{~cm}$ from the ileocecal junction. The mesentery was separated, intestinal fecal material was removed and then the tissue was rinsed with PBS three times. The samples $2 \mathrm{~cm}$ from the ileocecal junction were fixed with $10 \%$ formaldehyde at room temperature for $48 \mathrm{~h}$, stained with hematoxylin (at room temperature for $5 \mathrm{~min}$ ) and eosin (at room temperature for $1 \mathrm{~min}$ ) (H\&E) and subsequently prepared for immunohistochemical staining. The tissues $5 \mathrm{~cm}$ from the ileocecal junction were prepared for western blot analysis. The tissues obtained $8 \mathrm{~cm}$ from the ileocecal junction, were stored at $-80^{\circ} \mathrm{C}$ until measurement of MPO and DAO activities. Tissues obtained $11 \mathrm{~cm}$ from the ileocecal junction were analyzed for water content.

Measuring the level of serum TNF- $\alpha, H M G B 1$ and ghrelin. TNF- $\alpha$ (cat.no.CSB-E11987r), HMGB1 (cat.no.CSB-E08224r) and ghrelin ELISA kits (cat. no. CSB-E09816r) were used for the measurements of serum TNF- $\alpha$, HMGB1 and ghrelin (Shanghai Westang Bio-tech Co., Ltd., Shanghai, China). Optical density (OD) was measured using a microplate reader (Multiskan EX, V. 2.3; Thermo Fisher Scientific, Inc., Waltham, MA, USA) at $450 \mathrm{~nm}$ within $30 \mathrm{~min}$. Serum TNF- $\alpha$, HMGB1 and ghrelin levels were then calculated according to the OD value and standard curves.

Measuring MPO and DAO activity in the bowel tissues. MPO and DAO activities were measured using detection MPO biochemical kits and DAO biochemical kits (Beijing ComWin Biotech Co., Ltd., Beijing, China). After $100 \mathrm{mg}$ of bowel tissue was cut into small sections, $1 \mathrm{ml} 0.5 \%$ hexadecyltrimethylammonium bromide (Beijing Solarbio Science \& Technology Co., Ltd., Beijing, China) was added, and the tissue was homogenized. The homogenate was then centrifuged at $1,520 \mathrm{x} \mathrm{g}$ at $4^{\circ} \mathrm{C}$ for $10 \mathrm{~min}$, and the supernatant was collected for the measurement of MPO activity. 
A further $200 \mathrm{mg}$ bowel tissue was cut into small sections, a 7-fold volume of phosphate-buffered saline (PBS; $0.1 \mathrm{~mol}$, $\mathrm{pH}=7.2$ ) was added, the tissue was homogenized, then centrifuged at $9,500 \mathrm{x}$ g at $4^{\circ} \mathrm{C}$ for $10 \mathrm{~min}$ and the supernatant was collected to measure DAO activity. OD was measured with a microplate reader at $450 \mathrm{~nm}$ within $30 \mathrm{~min}$ of each assay and the activities of MPO and DAO in the bowel tissues were estimated using standard curves.

Measuring water content in bowel tissues. Water content in bowel tissues was measured using the wet-dry weighting method. In brief, fresh bowel tissue was obtained and blotting paper was used to remove surface water. The specimen was weighed on an electronic analytical balance $(1 / 100,000 \mathrm{~g})$ and this was recorded as the wet weight. The specimen was then placed in a draught drying cabinet and dried at $90^{\circ} \mathrm{C}$ for $72 \mathrm{~h}$ and weighed again; recorded as the dry weight. Water content (\%) was measured as (wet weight - dry weight) / wet weight x $100 \%$.

Pathological changes of the bowel tissues. Intestinal mucosa injury was scored using the Chiu method as reported by Bao et al (15) to grade the severity of the intestinal mucosa injury. Once the bowel tissue was obtained, the ileal tissues were fixed with $10 \%$ formaldehyde at $25^{\circ} \mathrm{C}$ for $48 \mathrm{~h}$, paraffin embedded, sliced and stained with H\&E. Subsequently, pathological changes in the intestinal tissues were evaluated and graded using the Chiu's score $(16,17)$. Chiu's score grading was as follows: Grade 0, normal mucosal villi; Grade 1, development of subepithelial Gruenhagen's space, usually observed at the apex of the villus, often with capillary congestion; Grade 2, extension of the subepithelial Gruenhagen's space with moderate lifting of epithelial layer from the lamina propria; Grade 3, massive epithelial lifting down the sides of villi, with a few tips being denuded; Grade 4; denuded villi with lamina propria and dilated capillaries exposed, increased cellularity of lamina propria; and Grade 5, digestion and disintegration of lamina propria, hemorrhage, and ulceration.

Measuring ghrelin and GSH-R protein expression in bowel tissues. Ghrelin, GSH-R and HMGB1 protein expression in bowel tissues were measured by western blot analysis. In brief, $20 \mathrm{mg}$ of the bowel tissues were cut into small sections, $200 \mu \mathrm{l}$ radioimmunoprecipitation assay buffer (Beyotime Institute of Biotechnology, Haimen, China; 50 mM TrisHCl pH 7.4, $1 \%$ NP-40, 0.5\% Na-deoxycholate, $0.1 \%$ SDS, $150 \mathrm{mM} \mathrm{NaCl}$, $2 \mathrm{mM}$ EDTA, $50 \mathrm{mM} \mathrm{NaF}$ ) was added to homogenize the tissues, and then they were centrifuged at $13,680 \mathrm{x}$ g at $4^{\circ} \mathrm{C}$ for $5 \mathrm{~min}$. The supernatant was collected and protein concentration was measured using the bicinchoninic acid (BCA) method (Beijing ComWin Biotech Co., Ltd.). Then, $30 \mu \mathrm{g}$ of the total protein underwent $10 \%$ SDS-PAGE, separated proteins were transferred to a nitrocellulose membrane (Beyotime Institute of Biotechnology) and blocked with $5 \%$ skimmed milk powder for $2 \mathrm{~h}$ at $25^{\circ} \mathrm{C}$. The membrane was then rinsed with Tris buffered saline, $0.05 \%$ Tween-20 (TBST) buffer 3 times. Ghrelin (cat. no. LS-C149239; 1:500, LifeSpan BioSciences, Inc., Seattle, WA, USA), GSH-R (cat. no. LS-C136920; 1:500, LifeSpan BioSciences, Inc.) or HMGB1 (cat. no. ab79823; 1:500; Abcam, Cambridge, UK) primary antibodies (1:500;
Abcam, Cambridge, UK) were added and incubated at $4{ }^{\circ} \mathrm{C}$ overnight. Then, horseradish peroxidase (HRP) labeled goat anti-rabbit secondary antibody (cat. no. CW0103s; 1:2,000; Beijing ComWin Biotech Co., Ltd.) was added and incubated at $25^{\circ} \mathrm{C}$ for $2 \mathrm{~h}$. ECL reagent (Beyotime Institute of Biotechnology) was then added according to the manufacturer's protocol. Antibodies against $\beta$-actin (cat. no. CW0096M; 1:2,000; Beyotime Institute of Biotechnology) were used as the internal reference. The Gel-Doc gel imaging system (Bio-Rad Laboratories, Inc., Hercules, CA, USA) was used to capture images and the VSD Gel Image Analysis System (Bio-Rad Laboratories, Inc.) was used for the semi-quantitative analysis of the bands. Relative protein expression was normalized to $\beta$-actin.

Immunohistochemistry. Ghrelin and GSH-R positive cells in the bowel tissues were measured by immunohistochemical examination. The tissues were fixed with $4 \%$ paraformaldehyde for $48 \mathrm{~h}$ at room temperature and then embedded in paraffin, sliced to a thickness of $4 \mu \mathrm{m}$ and placed on slides covered with polylysine. They were deparaffinized with dimethyl benzene, followed by gradient alcohol dehydration. Then, tissues were rinsed with $1 \mathrm{x}$ PBS (5 min $\mathrm{x} 3$ ), underwent 10 min incubation at $25^{\circ} \mathrm{C}$ with $3 \% \mathrm{H}_{2} \mathrm{O}_{2}$ and a further 3 rinses with $1 x$ PBS $(5 \mathrm{~min})$ were performed. Samples were blocked with $10 \%$ fetal bovine serum (FBS; Beijing ComWin Biotech Co., Ltd.) for $30 \mathrm{~min}$ at $25^{\circ} \mathrm{C}$ and then ghrelin (cat. no. LS-C149239) or GSH-R antibodies (cat. no. LS-C136920; both 1:100; both LifeSpan BioSciences, Inc.) were added and incubated overnight at $4^{\circ} \mathrm{C}$. The slides were then washed with 1xPBS (5 min x3). HRP labeled secondary antibody (cat. no. CW0103s; 1:200; Beijing ComWin Biotech Co., Ltd.) was added followed by incubation for $1 \mathrm{~h}$ at $25^{\circ} \mathrm{C}$ and another 3 washes with PBS (5 min) were performed. Staining with 3,3'-Diaminobenzidine and hematoxylin was performed. The section was then cleared with dimethyl benzene (30 min $\mathrm{x} 2$ ) and mounted with neutral resins, prior to observation and imaging with a CP62-JSM-6390LV microscope (Midwest Technology Co., Ltd., Beijing, China). Images were analyzed with Image-Pro Plus image analysis software (Media Cybernetics, Version 4.1, USA). For each slide, 10 non-overlapping vision fields were selected under magnification: $\mathrm{x} 400$, then the cumulative integrated optical density and the area of detection in each visual field was recorded under the same light intensity.

Statistical analysis. SPSS 17.0 software (SPSS Inc., Chicago, IL, USA) was used for the statistical analysis. Quantitative data are presented as the mean \pm standard deviation. Statistical significance was evaluated by independent sample t-test or one-way analysis of variance with least significant difference (LSD) test for post hoc analysis. Chiu's scoring $(16,17)$ was presented as median \pm interquartile Range (IQR), and analyzed by Kruskal-Wallis $\mathrm{H}$ test. $\mathrm{P}<0.05$ was considered to indicate a statistically significant difference.

\section{Results}

Electroacupuncture promotes ghrelin and GSH-R expression in rats with ABI. Compared with rats in the Sham group, ABI 
rats had $85 \%$ of the level of serum ghrelin, $(86.7 \pm 6.4 \mathrm{ng} / \mathrm{ml}$ vs. $102.4 \pm 4.9 \mathrm{ng} / \mathrm{ml}), 53 \%$ of the bowel ghrelin level $(0.08 \pm 0.01$ vs. $0.15 \pm 0.01)$ and $30 \%$ of the GSH-R level $(0.03 \pm 0.01$ vs. $0.10 \pm 0.06$; all $\mathrm{P}<0.05$; Fig. $1 \mathrm{~A}$ and $\mathrm{B}$ ). In ABI+EA rats, serum ghrelin, bowel ghrelin and GSH-R were all significantly higher than in the ABI group $(\mathrm{P}<0.05)$. Compared with rats in the Sham group, ABI+GHRA+EA rats had $89 \%$ of the serum ghrelin levels $(91.3 \pm 6.0 \mathrm{ng} / \mathrm{ml}), 53 \%$ of the bowel ghrelin $(0.08 \pm 0.01)$ and $20 \%$ of the GSH-R levels $(0.02 \pm 0.00$; all $\mathrm{P}<0.05$; Fig. $1 \mathrm{~A}$ and $\mathrm{B})$.

The level of serum ghrelin in bowel tissue, determined by western blot analysis, was supported by the immunohistochemistry results for ghrelin and GSH-R (Fig. 2).

Electroacupuncture reduces serum TNF- $\alpha$ and HMGB1 levels by promoting ghrelin secretion in rats with $A B I$. Serum TNF- $\alpha$ and HMGB1 levels in each group are presented in Fig. 3. Compared with the Sham group, rats in the ABI group exhibited a significant $226 \%$ increase in the level of serum TNF- $\alpha$ $(164.9 \pm 60.3 \mathrm{pg} / \mathrm{ml}$ vs. $72.9 \pm 15.8 \mathrm{pg} / \mathrm{ml} ; \mathrm{P}<0.05$; Fig. $3 \mathrm{~A})$, a significant $258 \%$ increase in the level of serum HMGB1 $(79.5 \pm 32.8 \mathrm{pg} / \mathrm{ml}$ vs. $30.8 \pm 8.7 \mathrm{pg} / \mathrm{ml} ; \mathrm{P}<0.05$; Fig. $3 \mathrm{~A}$ ) and a significant $210 \%$ increase in the level of HMGB1 in bowel tissue $(0.44 \pm 0.10$ vs. $0.21 \pm 0.07 ; \mathrm{P}<0.05$; Fig. $3 \mathrm{~B})$. Compared with $\mathrm{ABI}$ rats, the $\mathrm{ABI}+\mathrm{EA}$ rats had significantly lower TNF- $\alpha$ levels $(105 \%$ of Sham, $76.8 \pm 16.6 \mathrm{pg} / \mathrm{ml}$; Fig. 3A), serum HMGB1 (104\% of Sham, 32.1 $\pm 10.8 \mathrm{pg} / \mathrm{ml}$; Fig. 3A) and bowel tissue HMGB1 (138\% of Sham, 0.29 \pm 0.08 ; Fig. 3B; all P<0.05). Compared with the ABI+EA group, the ABI+GHRA+EA group had significantly higher TNF- $\alpha$ levels (173\% of Sham, $126.1 \pm 19.6 \mathrm{pg} / \mathrm{ml} ; \mathrm{P}<0.05$; Fig. 3A) and bowel tissue HMGB1 (214\% of Sham, $0.45 \pm 0.06 ; \mathrm{P}<0.05$; Fig. 3B). There were also higher levels of serum HMGB1 in this group (156\% of Sham, $48.1 \pm 16.5 \mathrm{pg} / \mathrm{ml}$; Fig. 3A), though this difference was not significant compared with the ABI+EA group.

Electroacupuncture decreased MPO activity but increased DAO activity in bowel tissues by promoting ghrelin secretion. The results of the MPO and DAO activity investigations are presented in Fig. 4. The ABI group exhibited a decrease in DAO activity compared with the Sham group $(13.52 \pm 2.33 \mathrm{U} / \mathrm{ml}$ vs. $18.74 \pm 4.18 \mathrm{U} / \mathrm{ml}$; Fig. $4 \mathrm{~A}$ ) and a $446 \%$ increase in MPO activity $(21.83 \pm 19.55 \mathrm{U} / \mathrm{ml}$ vs. $4.89 \pm 1.68 \mathrm{U} / \mathrm{ml}$; Fig. 4B; both $\mathrm{P}<0.05)$. Compared with the ABI group, DAO activity in the ABI+EA group increased back to levels similar to the Sham group $(18.74 \pm 4.18 \mathrm{U} / \mathrm{ml} ; \mathrm{P}<0.05$; Fig. $4 \mathrm{~A})$, whereas MPO activity significantly decreased, to $140 \%$ of the Sham group $(6.89 \pm 2.05 \mathrm{U} / \mathrm{ml})$ compared with the ABI group $(\mathrm{P}<0.05$; Fig. 4B). In the ABI+GHRA+EA group, DAO activity was $82 \%$ of the Sham group $(15.38 \pm 2.69 \mathrm{U} / \mathrm{ml})$ and MPO activity was $227 \%$ of the Sham group value $(11.09 \pm 3 \mathrm{U} / \mathrm{ml})$.

Electroacupuncture improved intestinal mucosal barrier and reduced water content in bowel tissues by promoting ghrelin secretion. The pathological changes in the bowel were analyzed histologically and representative samples are presented in Fig. 5A. The following characteristics were observed: In the Sham group, the villus was neat, perivascular structurally normal, there was no evidence of significant bleeding, the muscle fibers were arranged in neat rows and
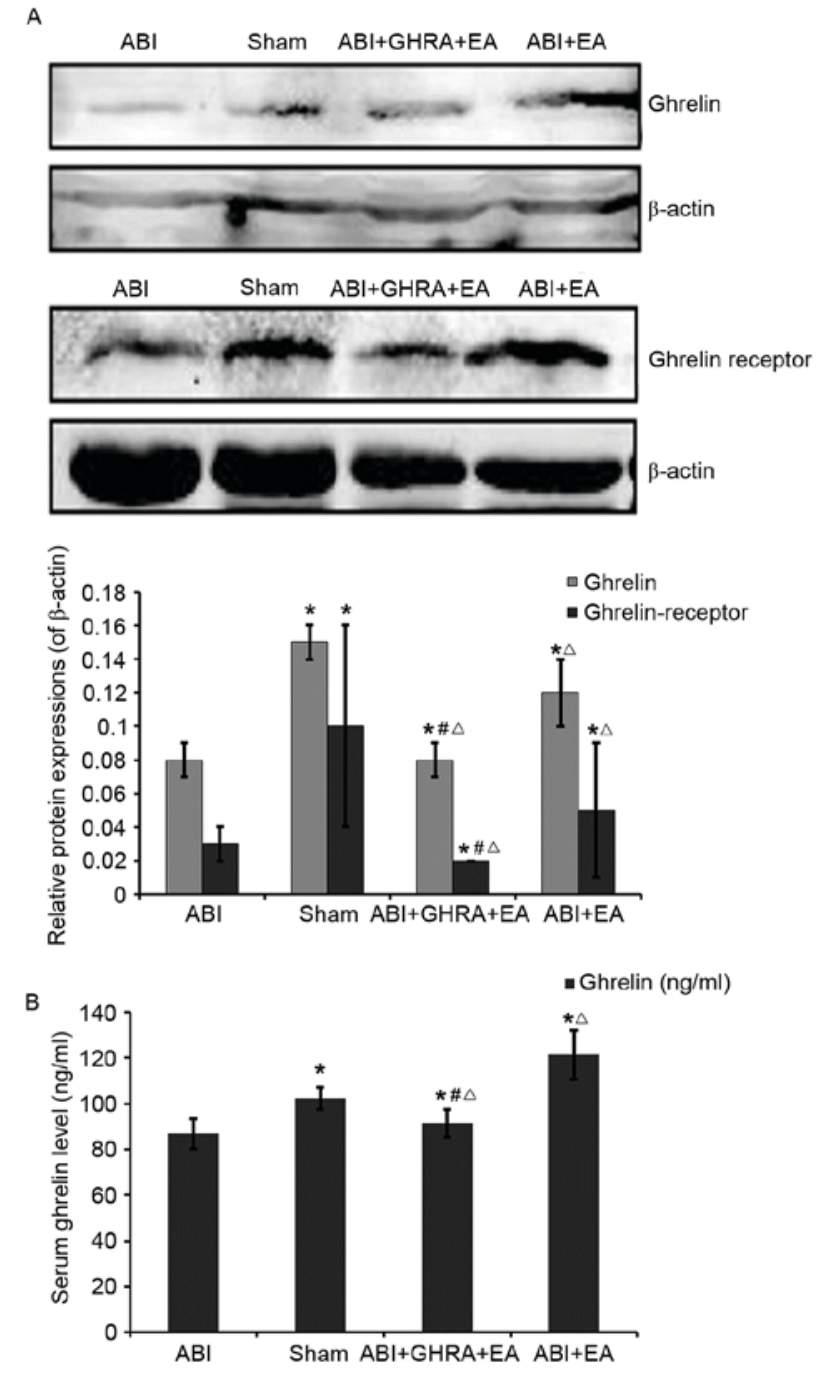

Figure 1. Effects of EA on the level of serum and protein GSH, GSH-R in the bowel tissues of ABI rat models. ABI rat models were induced by CLP. For rats in the ABI+EA group, EA was performed for $30 \mathrm{~min}, 20 \mathrm{~min}$ after CLP. For rats in the ABI+GHRA+EA group, a microinjection pump was used to inject GHRA for $1 \mathrm{~h}$ via the tail vein following CLP but prior to EA. (A) Protein expression of ghrelin and its receptor were determined by western blot analysis. $\beta$-actin was used as an internal control. (B) The level of serum ghrelin was determined by ELISA. ${ }^{\mathrm{P}}<0.05$ vs. ABI group; ${ }^{\wedge} \mathrm{P}<0.05$ vs. Sham group; " $\mathrm{P}<0.05$ vs. ABI+EA group. Data are presented as mean \pm standard deviation ( $\mathrm{n}=12$ each group). EA, electroacupuncture; $\mathrm{ABI}$, acute bowel injury; CLP, cecal ligation and puncture; GHRA, GSH-R Blocker; GSH, ghrelin; GSH-R, ghrelin receptor.

the serosa was normal. In the ABI group, there was intestinal villi damage, significant swelling, ulcers, clear perivascular bleeding, neutrophil infiltration and basal fractures. In the ABI+EA group, a small amount of intestinal villi defects, a small amount of necrosis, a small amount of bleeding and a small amount of neutrophil infiltration was detected. In the ABI+GHRA+EA group, there were clearly damaged intestinal villi and swelling, accompanied by bleeding and ulceration.

Pathological changes in the intestinal tissues were evaluated according to the method reported by Chiu et al (16) (Table I). Chui's score (median \pm interquartile range) in the Sham, ABI, ABI+EA and ABI+GHRA+EA groups were 0 , $4 \pm 0,2 \pm 1$ and $4 \pm 0$, respectively. Chiu's score in the ABI+EA group was significantly lower than that of the ABI group 
A
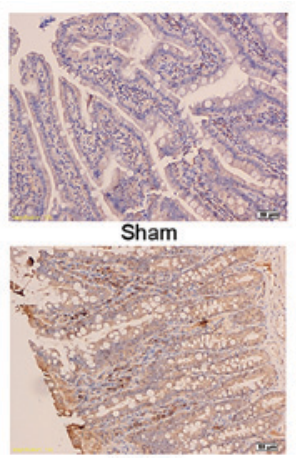

$\mathrm{ABI}+\mathrm{EA}$

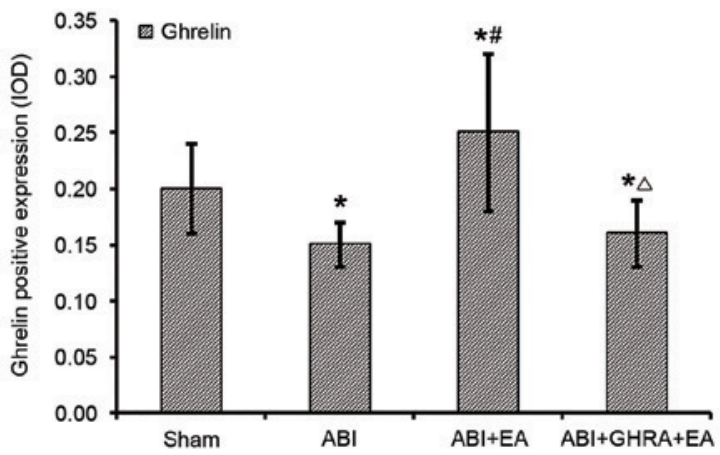

B

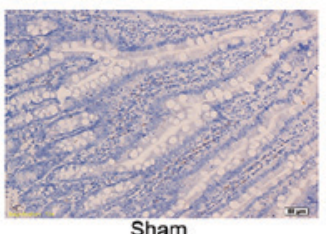

$\mathrm{ABI}$

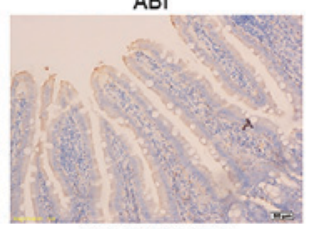

$\mathrm{ABI}+\mathrm{GHRA}+\mathrm{EA}$

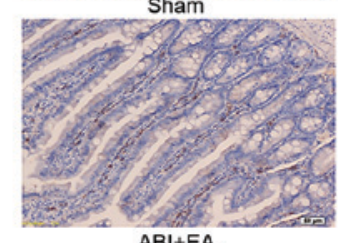

$A B I+E A$

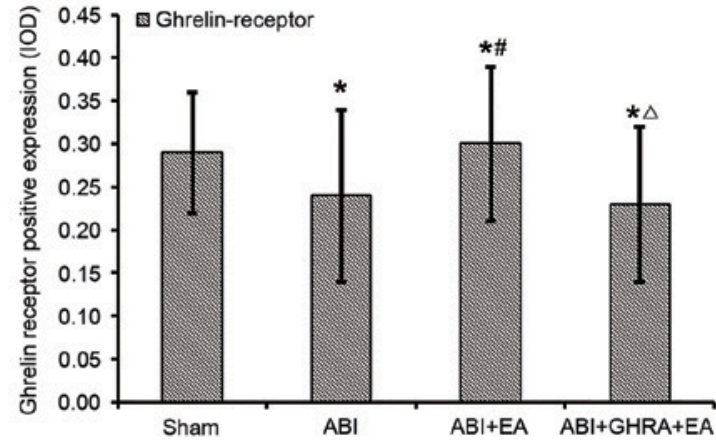

Figure 2. EA promoted the expression of ghrelin and its receptor in the bowel tissues of ABI rat models. Protein expression of (A) ghrelin and (B) its receptor were determined by immunohistochemistry (Magnification, $\mathrm{x} 400$ ). ${ }^{*} \mathrm{P}<0.05$ vs. sham group; ${ }^{\sharp} \mathrm{P}<0.05$ vs. ABI group; ${ }^{\triangle} \mathrm{P}<0.05$ vs. ABI+EA group. Data are presented as mean \pm standard deviation ( $\mathrm{n}=12$ each group). ABI, acute bowel injury; EA, electroacupuncture; GHRA, ghrelin receptor blocker; IOD, integrated optical density.

A
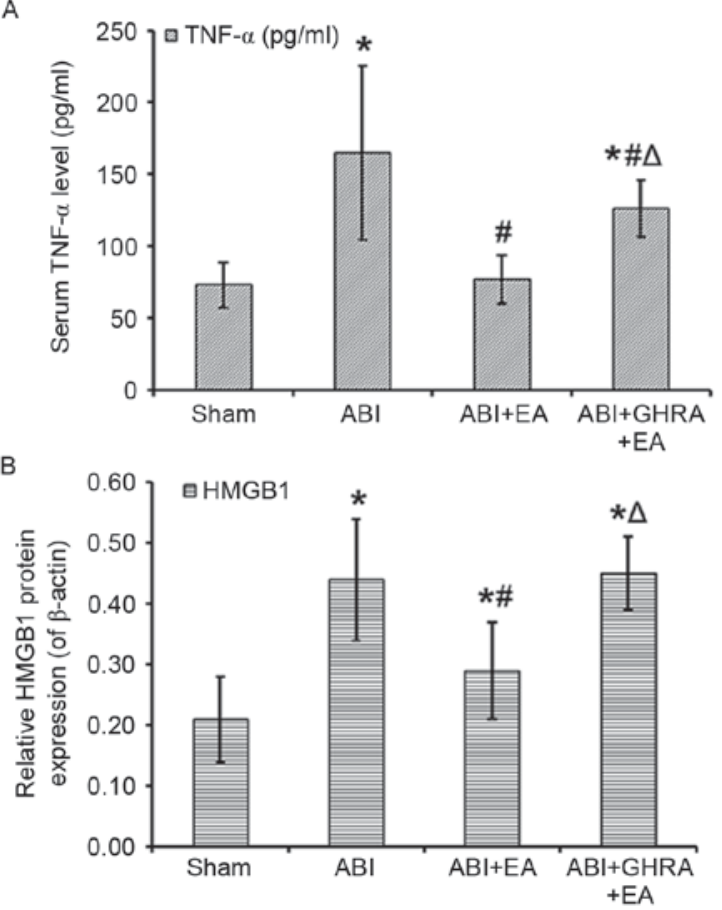

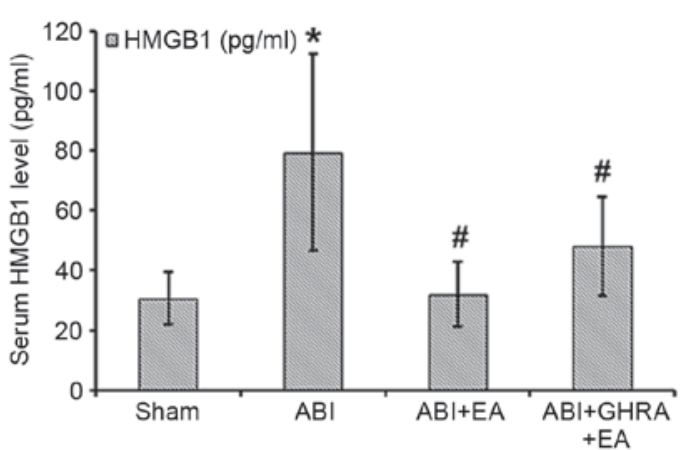

HMGB1

$\beta$-actin
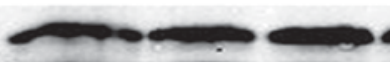

Sham

$\mathrm{ABI}$

$A B|+E A \quad A B|+G H R A$

$+\mathrm{EA}$

Figure 3. EA reduced the level of TNF- $\alpha$ and HMGB1 in the serum and the level of HMGB1 in bowel tissues by promoting ghrelin secretion in ABI rat models. (A) Serum TNF- $\alpha$ and HMGB1 levels, as determined by ELISA. (B) Protein expression of HMGB1 in bowel tissue as determined by western blot analysis. ${ }^{*} \mathrm{P}<0.05$ vs. sham group; ${ }^{\#} \mathrm{P}<0.05$ vs. ABI group; ${ }^{\Delta} \mathrm{P}<0.05$ vs. ABI+EA group. Data are presented as mean \pm standard deviation $(\mathrm{n}=12$ each group). EA, electroacupuncture; TNF- $\alpha$, tumor necrosis factor- $\alpha$; HMB1, high mobility group box 1; ABI, acute bowel injury; GHRA, ghrelin receptor blocker.

$(\mathrm{P}<0.05)$, however Chiu's score in the $\mathrm{ABI}+\mathrm{GHRA}+\mathrm{EA}$ group was significantly higher than that of the ABI+EA group $(\mathrm{P}<0.05)$.
Bowel water content was also measured. Water content in the ABI group was significantly increased by $121 \%$ compared with the Sham group $(79.42 \pm 7.36 \%$ vs. $65.55 \pm 7.37 \%$; $\mathrm{P}<0.05)$. 


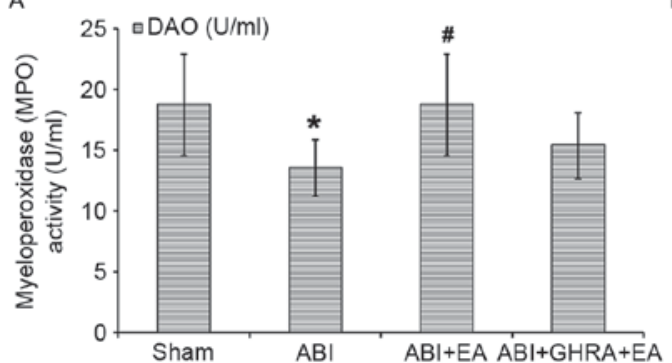

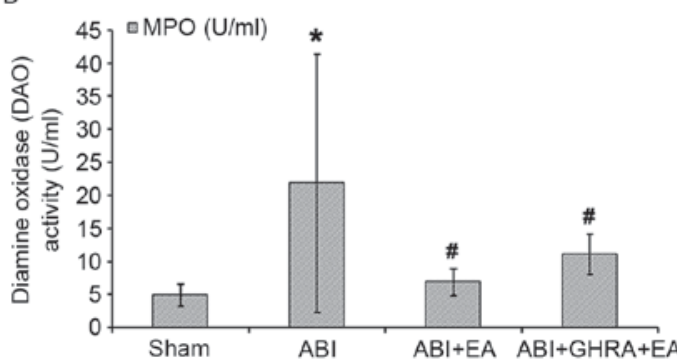

Figure 4. EA reduced (A) MPO activity but increased (B) DAO activity in bowel tissues by promoting ghrelin secretion in $\mathrm{ABI}$ rat models. "P<0.05 vs. sham group; ${ }^{\prime} \mathrm{P}<0.05$ vs. ABI group. Data are presented as mean \pm standard deviation ( $\mathrm{n}=12$ each group). EA, electroacupuncture; MPO, myeloperoxidase; DAO, diamine oxidase; ABI, acute bowel injury; GHRA, ghrelin receptor blocker.
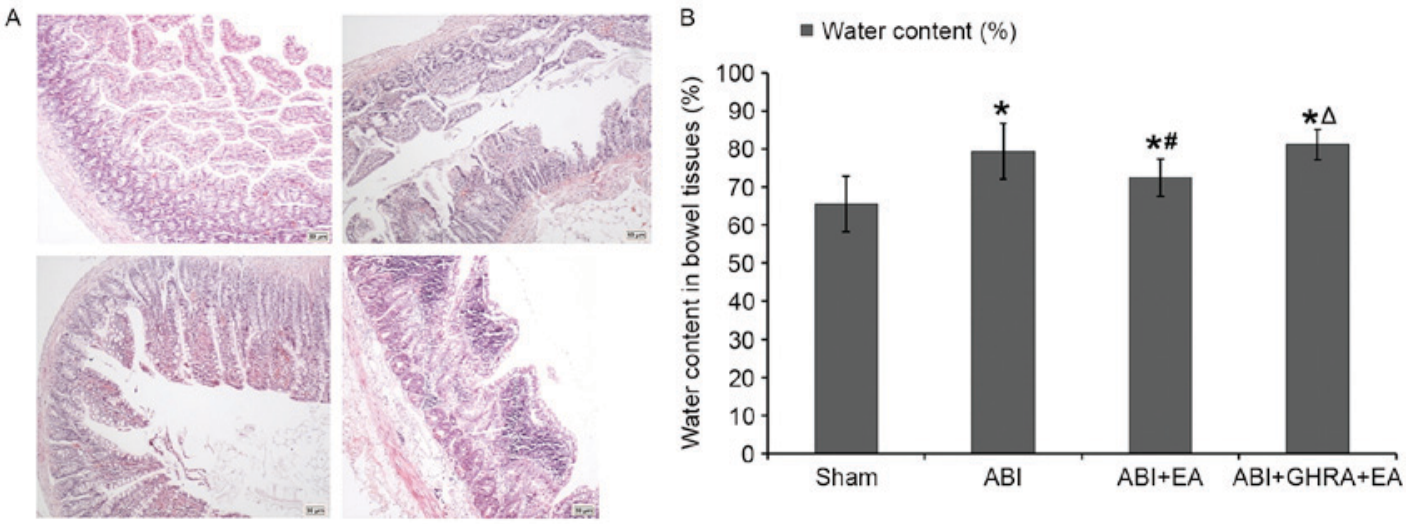

Figure 5. EA improved intestinal mucosal barrier and reduced water content in bowel tissues by promoting ghrelin secretion in ABI rat models. (A) Hematoxylin and eosin staining was used to detect pathological changes (Magnification, x100); pathological changes in the intestinal tissues were evaluated according to the method reported by Chiu et al, presented in Table I. (B) Measuring water content in bowel tissues. ${ }^{~} \mathrm{P}<0.05$ vs. sham group; ${ }^{*} \mathrm{P}<0.05$ vs. ABI group; ${ }^{\wedge} \mathrm{P}<0.05$ vs. ABI+EA group. Data are presented as mean \pm standard deviation ( $\mathrm{n}=12$ each group). EA, electroacupuncture; ABI, acute bowel injury; GHRA, ghrelin receptor blocker.

The water content in the ABI+EA group was significantly reduced compared with the ABI group $(72.41 \pm 4.89 \%$; $\mathrm{P}<0.05)$ and the water content in the $\mathrm{ABI}+\mathrm{GHRA}+\mathrm{EA}$ group was significantly greater than that of the ABI+EA group (81.13 $\pm 3.94 \%$; $\mathrm{P}<0.05$; Fig. 5B).

\section{Discussion}

The aim of the present study was to investigate the role of electroacupuncture in assisting the recovery of rats from ABI and determine whether ghrelin was involved in the recovery process. The results of the current study suggest that electroacupuncture promoted ghrelin and GSH-R expression and decreased the inflammatory response, indicated by lower levels of the pro-inflammatory factors TNF- $\alpha$ and HMGB1 following electroacupuncture. The function of the gastrointestinal barrier was also improved following electroacupuncture, suggested by the restoration of DAO activity levels and the decrease in MPO activity towards levels observed in the Sham group. The recovery of rats following ABI by electroacupuncture was also inhibited by GSH-R.

The inflammatory response that culminates in MODS is mediated by a complex interaction of pro-inflammatory factors called cytokines, two of which are TNF- $\alpha$ and HMGB1 (18). It has been suggested that HMGB1 may be a marker of subclinical intestinal inflammation (19). A potential marker for intestinal damage is DAO (20), which is primarily expressed in the small intestine and its expression and activity decrease following intestinal injury. The opposite is observed with MPO, a peroxidase enzyme identified primarily in neutrophils, as its activity increases as a result of neutrophil infiltration of the intestine (21). The present study revealed that electroacupuncture reversed the increase in TNF- $\alpha$ and HMGB1 levels in rats that had undergone ABI and improved the function of the intestinal mucosal barrier, indicated by the restoration of DAO activity levels and the decrease in MPO towards levels observed in the Sham group. These results for TNF- $\alpha$ and improved function of the intestinal mucosal barrier have been observed in a previous clinical study (7), and similar results have also been obtained in previous studies investigating the liver, kidney and jejunum tissues in CLP rats $(6,22)$. Electroacupuncture significantly inhibits TNF- $\alpha$ levels in the liver, kidney and jejunum tissues in ABI rat models. Electroacupuncture improves blood flow and perfusion of the tissues, thus reducing organ edema and dysfunction (6). Electroacupuncture applied to the bilateral ST-25 and ST-36 in an acute pancreatitis rat model also demonstrated an improved intestinal propulsion rate, reduced pro-inflammatory markers and improved acute lung injury (22). Acupuncture may not only inhibit the release of pro-inflammatory factors but also increase the secretion of gastrointestinal hormones. Wu et al (23), assessed the effects of electroacupuncture on mast cells, substance P (SP) and 
Table I. EA improved intestinal mucosal barrier in bowel tissues by promoting ghrelin secretion in ABI rat models.

\section{Groups}

\section{Sham}

ABI

$\mathrm{ABI}+\mathrm{EA}$

$\mathrm{ABI}+\mathrm{GHRA}+\mathrm{EA}$

Chiu's score (16)

Data are presented as median \pm interquartile Range $(\mathrm{n}=12$ each group). ${ }^{\mathrm{a}} \mathrm{P}<0.05$ vs. sham group; ${ }^{\mathrm{b}} \mathrm{P}<0.05$ vs. $\mathrm{ABI}$ group; ${ }^{\mathrm{c}} \mathrm{P}<0.05$ vs. ABI+EA group. ABI, acute bowel injury; EA, electroacupuncture; GHRA, ghrelin receptor blocker.

vasoactive intestinal peptide (VIP) in rat models of irritable bowel syndrome, and revealed that the sensitivity threshold in the rat models was significantly lower than the rats in the control group, whereas the mast cell count and secretion of SP and VIP were significantly higher. Lin et al (24) indicated that acupuncture on Zusanli acupoints may improve gastric motility, blood flow of gastric mucosa and the secretion of gastrointestinal hormones, including motilin.

The present study demonstrated that ghrelin and GSH-R levels decreased in the rats with ABI, in accordance with previous findings $(25,26)$. Das et al $(8)$ revealed that the level of ghrelin decreased significantly with the progression of sepsis, aggravation of gastrointestinal dysfunction and occurrence of gastric secretion disorders. Another study demonstrated that ghrelin was associated with the prognosis of patients with sepsis and that ghrelin levels were significantly higher in patients that survived compared with those that succumbed (12). In addition, levels of ghrelin and GSH-R were closely associated with impaired gastrointestinal mucosal function and the severity of this impairment (27). In animal models of acute renal injury, induced by intraperitoneal injection of lipopolysaccharide, an injection of ghrelin either prior to or following injury reduced serum levels of TNF-a, interleukin (IL)-1 $\beta$, and IL-6, thus partially restoring the impaired glomerular filtration rate (28). Administration of exogenous ghrelin also significantly increased the degree of vascular relaxation and inhibited the nuclear factor $-\kappa \mathrm{B}(\mathrm{NF}-\kappa \mathrm{B})$ pathway. This improved tissue perfusion and the permeability of the intestinal mucosa, inhibited the production of inflammatory mediators, reduced intestinal wall edema, decreased bacterial translocation, delayed sepsis progress into shock and finally served important roles in improving septic intestinal dysfunction in rat models of sepsis (29). In rat models of acute pulmonary injuries induced by CLP, ghrelin increased the blood flow in the lungs, inhibited the activation of NF- $\mathrm{NB}$, down-regulated pro-inflammatory factors, decreased the damage to the pulmonary tissues, and thus greatly reduced the mortality of the rats (30).

In addition, the present study demonstrated that electroacupuncture promoted the expression of ghrelin and GSH-R in rats with $\mathrm{ABI}$. To the best of our knowledge, this is the first study to identify the effects of electroacupuncture on the increased secretion of ghrelin and GSH-R. GSH-R blocker was administered to the rats following CLP but prior to the stimulation of electroacupuncture. The protective effects of electroacupuncture on the intestinal mucosal barrier were reversed and there was an increase in serum TNF- $\alpha$ and HMGB1 levels, suggesting that the effects of Zusanli acupoints electroacupuncture on ABI are associated with ghrelin and GSH-R levels. Therefore, electroacupuncture on Zusanli acupoints may increase ghrelin levels in the body by increasing the expression of ghrelin and GSH-R in bowel tissues. Ghrelin may, in turn, inhibit the release of pro-inflammatory factors, improve the perfusion of bowel tissues and protect the bowel mucosal barrier. Future studies are required to investigate the detailed mechanisms involved in increasing ghrelin and GSH-R expression by Zusanli acupoint electroacupuncture in rats with ABI. Electroacupuncture ameliorated ABI in rats by promoting the secretion of ghrelin and upregulating the expression of GSH-R. This information may assist with the use of electroacupunture as a clinical therapy for ABI in the early treatment of sepsis.

\section{Acknowledgements}

The present study was supported by Zhejiang provincal key program for traditional Chinese medicine in prevention and treatment of major diseases (grant no. 2012ZGG001).

\section{References}

1. Malbrain ML and De Laet I: AIDS is coming to your ICU: Be prepared for acute bowel injury and acute intestinal distress syndrome. Intensive Care Med 34: 1565-1569, 2008.

2. De-Souza DA and Greene LJ: Intestinal permeability and systemic infections in critically ill patients: Effect of glutamine. Crit Care Med 33: 1125-1135, 2005.

3. Seoane L, Winterbottom F, Nash T, Behrhorst J, Chacko E, Shum L, Pavlov A, Briski D, Thibeau S, Bergeron D, Rafael T and Sundell E: Using quality improvement principles to improve the care of patients with severe sepsis and septic shock. Ochsner J 13: 359-366, 2013.

4. Hu S, Song Q, Wang HB, Lü Y, Yu Y, Wang L, Zhou GY, Shi X and Sheng ZY: Study on the protective effect and mechanism of electroacupuncturing at Zusanli point (ST 36) on endotoxin induced hepatic injury in rats. Zhong Guo Zhong Xi Yi Jie He Ji Jiu Za Zhi Bian Ji Bu 14: 296-298, 2007 (In Chinese).

5. Song Q, Hu S, Wang H, Lv Y, Shi X, Sheng Z and Sheng W: Electroacupuncturing at Zusanli point (ST36) attenuates pro-inflammatory cytokine release and organ dysfunction by activating cholinergic anti-inflammatory pathway in rat with endotoxin challenge. Afr J Tradit Complement Altern Med 11: 469-474, 2014.

6. Song XM, Li JG, Wang YL, Hu ZF, Zhou Q, Du ZH and Jia BH: The protective effect of the cholinergic anti-inflammatory pathway against septic shock in rats. Shock 30: 468-472, 2008.

7. Wang LC and Wu JN: The clinical efficacy of sepsis with early intervention of electroacupuncture. Life Sci J 10: 1900-1903, 2013.

8. Das UN: Relationship between gut and sepsis: Role of ghrelin. World J Diabetes 2: 1-7, 2011.

9. Wu R, Dong W, Qiang X, Wang H, Blau SA, Ravikumar TS and Wang P: Orexigenic hormone ghrelin ameliorates gut barrier dysfunction in sepsis in rats. Crit Care Med 37: 2421-2426, 2009.

10. Kojima $\mathbf{M}$ and Kangawa K: Ghrelin: Structure and function. Physiol Rev 85: 495-522, 2005.

11. Li WG, Gavrila D, Liu X, Wang L, Gunnlaugsson S, Stoll LL, McCormick ML, Sigmund CD, Tang C and Weintraub NL: Ghrelin inhibits proinflammatory responses and nuclear factor-kappaB activation in human endothelial cells. Circulation 109: 2221-2226, 2004.

12. Koch A, Sanson E, Helm A, Voigt S, Trautwein C and Tacke F: Regulation and prognostic relevance of serum ghrelin concentrations in critical illness and sepsis. Crit Care 14: R94, 2010. 
13. Chaudry IH, Tabata Y, Schleck S and Baue AE: Effect of splenectomy on reticuloendothelial function and survival following sepsis. J Trauma 20: 649-656, 1980.

14. Rittirsch D, Huber-Lang MS, Flierl MA and Ward PA: Immunodesign of experimental sepsis by cecal ligation and puncture. Nat Protoc 4: 31-36, 2009.

15. Bao J, Tan S, Yu W, Lin Z, Dong Y, Chen Q, Shi J, Duan K, Bai X, Xu L, et al: The effect of peritoneal air exposure on intestinal mucosal barrier. Gastroenterol Res Pract 2014: 674875, 2014.

16. Chiu CJ, Mcardle AH, Brown R, Scott HJ and Gurd FN: Intestinal mucosal lesions in low flow states. I. A morphological, hemodynamic and metabolic reappraisal. Arch Surg 101: 478-483, 1970

17. Longa EZ, Weinstein PR, Carlson S and Cummins R: Reversible middle cerebral artery occlusion without craniectomy in rats. Stroke 20: 84-91, 1989.

18. Surbatovic M, Veljovic M, Jevdjic J, Popovic N, Djordjevic D and Radakovic S: Immunoinflammatory response in critically ill patients: Severe sepsis and/or trauma. Mediators Inflamm 2013: 362793, 2013.

19. Palone F, Vitali R, Cucchiara S, Pierdomenico M, Negroni A Aloi M, Nuti F, Felice C, Armuzzi A and Stronati L: Role of HMGB1 as a suitable biomarker of subclinical intestinal inflammation and mucosal healing in patients with inflammatory bowel disease. Inflamm Bowel Dis 20: 1448-1457, 2014.

20. Zhao L, Luo L, Jia W, Xiao J, Huang G, Tian G, Li J and Xiao Y: Serum diamine oxidase as a hemorrhagic shock biomarker in a rabbit model. PLoS One 9: e102285, 2014.

21. Mondello S, Galuppo M, Mazzon E, Domenico I, Mondello P, Carmela A and Cuzzocrea S: Glutamine treatment attenuates the development of ischaemia/reperfusion injury of the gut. Eur J Pharmacol 643: 304-315, 2010
22. Guo H, Zhu SF, Zhang RR, Zhao XL, Wan MH and Tang WF: Electroacupuncture ameliorates acute lung injury through promoting gastrointestinal motility in rats with acute pancreatitis. Evid Based Complement Alternat Med 2014: 943596, 2014

23. Wu HG, Jiang B, Zhou EH, Shi Z, Shi DR, Cui YH, Kou ST and Liu HR: Regulatory mechanism of electroacupuncture in irritable bowel syndrome: Preventing MC activation and decreasing SP VIP secretion. Dig Dis Sci 53: 1644-1651, 2008.

24. Lin YP, Yi SX, Yan J and Chang XR: Effect of acupuncture at Foot-Yangming Meridian on gastric mucosal blood flow, gastric motility and brain-gut peptide. World J Gastroenterol 13 2229-2233, 2007

25. Cheyuo C, Jacob A and Wang P: Ghrelin-mediated sympathoinhibition and suppression of inflammation in sepsis. Am J Physio Endocrinol Metab 302: E265-E272, 2012.

26. Jacob A, Wu R, Zhou M, Coppa GF and Wang P: Mechanism of the inhibitory effect of ghrelin in sepsis. Hepat Med 2: 33-38, 2010.

27. Serpa Neto A, Veelo DP, Peireira VG, de Assunção MS Manetta JA, Espósito DC and Schultz MJ: Fluid resuscitation with hydroxyethyl starches in patients with sepsis is associated with an increased incidence of acute kidney injury and use of renal replacement therapy: A systematic review and meta-analysis of the literature. J Crit Care: 185.e1-7, 2014.

28. Arnes L, Hill JT, Gross S, Magnuson MA and Sussel L: Ghrelin expression in the mouse pancreas defines a unique multipotent progenitor population. PLoS One 7: e52026, 2012.

29. Wu R, Dong W, Zhou M, Cui X, Hank Simms H and Wang P: Ghrelin improves tissue perfusion in severe sepsis via downregulation of endothelin-1. Cardiovasc Res 68: 318-326, 2005.

30. Wu R, Dong W, Zhou M, Zhang F, Marini CP, Ravikumar TS and Wang P: Ghrelin attenuates sepsis-induced acute lung injury and mortality in rats. Am J Respir Crit Care Med 176: 805-813, 2007. 